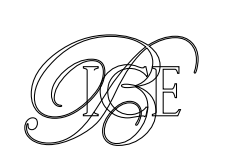

Fernando González*

Diego Martínez López ${ }^{* *}$

\title{
EL DISEÑO DE REGLAS FISCALES EN GOBIERNOS SUBCENTRALES. EL CASO DE ESPAÑA
}

La crisis de la COVID-19 ha confirmado la necesidad de repensar las actuales reglas fiscales. Por su parte, la literatura ha prestado poca atención a las reglas fiscales aplicables a Gobiernos subnacionales (GSN). En este artículo se presenta el marco actual de reglas fiscales en los GSN en un país altamente descentralizado, como es España, los principales criterios a discutir en el diseño de las mismas y las líneas de reforma que podrían adoptarse, teniendo en cuenta los nuevos desafíos originados con la pandemia.

Palabras clave: reglas fiscales, déficit público, deuda pública, comunidades autónomas, corporaciones locales.

Clasificación JEL: H70, H74.

\section{Introducción}

Contrariamente a una tendencia actual de opinión, la pandemia COVID-19 no ha supuesto el fin de la necesidad de contar con reglas fiscales. Más bien al contrario: el nivel de gasto inesperado y los consecuentes niveles de endeudamiento justifican, si cabe todavía más, la necesidad de contar con un marco de reglas fiscales eficaz, flexible, simple y que tenga como objetivo el control de la deuda.

Las reglas fiscales pueden ser definidas como límites numéricos fijos (suelos o techos)

\footnotetext{
* Técnico Comercial y Economista del Estado.

** Universidad Pablo Olavide de Sevilla.

Versión de enero de 2021.

Nota de los autores: todas las opiniones, errores u omisiones son de nuestra exclusiva responsabilidad personal, sin coincidir necesariamente con las de las instituciones a las que pertenecemos.

DOI: https:/doi.org/10.32796/bice.2021.3133.7168
}

aplicados a las variables fiscales a través de la legislación y vinculantes durante periodos plurianuales (Eyraud et al., 2020). Se trata, por tanto, de elementos de disciplina sobre la gestión de la política fiscal nacional y la de los Gobiernos subnacionales (GSN). En este último caso, la imposición de reglas fiscales viene justificada, además, por las mayores posibilidades de desembocar en episodios de bail-out, pudiendo ocasionar grandes costes económicos y sociales, no solo en la jurisdicción en cuestión, sino en todo el país.

Desde el punto de vista teórico existe una extensa literatura relativa al diseño de reglas fiscales para Gobiernos centrales, pero no se encuentran muchas guías normativas para el caso específico de las reglas fiscales en los GSN —quizás Eyraud et al. (2020) sea una de las excepciones-. El diseño de reglas fiscales en $D$ 
los GSN plantea problemas específicos que lo diferencian del diseño para Gobiernos nacionales. Por un lado, el sesgo hacia el déficit puede ser mayor en los GSN que en Gobiernos nacionales. Por otro lado, es necesario tener en cuenta las importantes competencias que pueden haber asumido los GSN y la inflexibilidad para adaptar sus presupuestos en función de la coyuntura económica, dificultando así el ejercicio de políticas fiscales contracíclicas.

En España, el proceso de descentralización comenzó ya hace más de tres décadas y, a día de hoy, casi dos tercios del gasto público total de las Administraciones (excluida la Seguridad Social) son realizados por los GSN. El caso español es una magnífica referencia para esta presentación: dos niveles de Gobierno subcentral con elevada potencia financiera (especialmente el regional), relativamente recientes reformas en la gobernanza fiscal del país que no terminan de ofrecer resultados aceptables, problemas de diseño y aplicación de incentivos correctamente alineados, etc. Recientemente, además, la supresión temporal de las reglas fiscales ha abierto el abanico de posibles enfoques.

Este artículo pretende valorar el marco español de reglas fiscales para GSN, trascurridos más de ocho años de vigencia desde su configuración actual en la Ley Orgánica de Estabilidad y Sostenibilidad Financiera (LOEPSF) ${ }^{1}$ y proponer alternativas en el diseño de las actuales reglas fiscales. La valoración que realizamos del marco actual viene guiada por los criterios señalados por organismos internacionales de referencia (European Fiscal Board, 2019), que adaptamos para su aplicación a un entorno descentralizado. Por su parte, prestamos una especial atención a los errores de diseño que, a nuestro juicio, sufre

Ley Orgánica 2/2012, de 27 de abril, de Estabilidad Presupuestaria y Sostenibilidad Financiera. el actual marco de gobernanza fiscal de nuestro país. Sobre esa base, proponemos a grandes rasgos dos diseños alternativos de reglas fiscales que ponen su foco en la reducción de la deuda pública, pero sin descuidar aspectos de estabilización económica.

La estructura de este artículo es la siguiente. En primer lugar, se describirá brevemente el marco actual de reglas fiscales aplicable en España a los GSN. Posteriormente, se debatirán sus resultados a partir de tres dimensiones: cómo ha contribuido a la mejora de las finanzas públicas, cuáles son sus propiedades anticíclicas y qué impacto ha tenido en la sostenibilidad de las finanzas públicas. A continuación se expondrán los principales problemas, a nuestro juicio, del diseño de este marco. Finalmente, se esbozarán dos propuestas para reformar el marco actual de reglas fiscales subnacionales. Un apartado de conclusiones cierra el trabajo.

\section{El marco común de reglas fiscales en España: la LOEPSF}

En este artículo adoptaremos una clasificación simplificada de Gobiernos subnacionales en comunidades autónomas (CC AA) y corporaciones locales (CC LL). La evolución de ambos niveles de gobierno ha sido diferente. Mientras que las CC LL han mantenido una cuota relativamente estable en el gasto total de las Administraciones públicas (AA PP) en torno al $16 \%$, las CC AA han incrementado su participación desde un $5 \%$ a comienzos de los años ochenta del siglo pasado hasta casi la mitad del gasto en la actualidad.

En la LOESPF se establecen tres reglas fiscales aplicables a los GSN, a saber: una regla de equilibrio estructural, un límite de deuda pública y una regla de gasto. Según la primera, $\triangleright$ 
ninguna Administración puede incurrir en déficit estructural (artículo 11.2). Esta regla, sin embargo, habría entrado en vigor a partir de 2020 de no haber mediado la pandemia. Hasta ese momento, al igual que ocurre con la deuda pública, rigen lo que la literatura denomina controles administrativos u objetivos fiscales, que, en puridad, no son reglas fiscales. El principio de estabilidad de la LOEPSF se evalúa en función del cumplimiento con un objetivo de déficit global, expresado en términos nominales, en relación al producto interior bruto (PIB) y establecido por el Consejo de Ministros para el año corriente y los tres siguientes. Para las CC LL hay obligación de encontrarse siempre en equilibrio o en superávit.

Según la regla de límite de deuda pública, esta no puede superar el $60 \%$ del PIB nacional. Este límite se distribuye por cada nivel de gobierno: $44 \%$ para el Estado, $13 \%$ para todas las CC AA (y también a nivel individual) y $3 \%$ para CC LL. Nuevamente estos límites operarían desde 2020 y, hasta ese momento, los objetivos de deuda plurianuales deberían haber asegurado la convergencia a esos niveles en 2020. Relacionado con esta regla, también se establece la prioridad absoluta del pago de la deuda pública: los intereses y el principal de la misma deben ser incluidos siempre en la formulación de los presupuestos y esas partidas no pueden ser modificadas a través del proceso presupuestario. La LOEPSF dispone, además, que cualquier superávit presupuestario debe ser destinado prioritariamente a la reducción del stock de deuda; por su parte, también se obliga a reducir el stock de deuda en caso de contar con ingresos imprevistos.

Según la regla de gasto, se limita el crecimiento del gasto computable de las AA PP al crecimiento de medio plazo del PIB. El gasto computable se define como los empleos no financieros, excluyendo los intereses de la deuda y otros gastos no discrecionales o financiados con transferencias de otras Administraciones. El gasto computable puede ser ajustado al alza o a la baja para reflejar los cambios normativos que supongan, respectivamente, aumentos o reducciones permanentes de recaudación.

Aparte de las reglas fiscales contenidas en la LOEPSF, también nos podemos referir a las reglas de oro que se aplican en las operaciones nuevas de endeudamiento de CC AA y CC LL. En términos generales, mientras que el recurso a la deuda de corto plazo se permite para cubrir desfases temporales de tesorería, el recurso al endeudamiento de largo plazo está restringido tanto para las CC AA como para las CC LL a la finalidad de operaciones de inversión, y para el caso de las CC AA, además, según la Ley Orgánica de Financiación de las Comunidades Autónomas (LOFCA), el servicio de la deuda no puede superar el $25 \%$ de sus ingresos corrientes ${ }^{2}$.

\section{Una valoración multidimensional}

Siguiendo los criterios de evaluación utilizados por el European Fiscal Board (2019), para analizar las reglas fiscales en la Unión Europea (UE) cabe realizar un ejercicio de valoración similar de las reglas españolas. Estos criterios se refieren a la posible mejora en la calidad de las finanzas públicas, a las propiedades de las reglas fiscales para la estabilización contracíclica de la economía y a su contribución a la sostenibilidad a largo plazo de $\triangle$

\footnotetext{
2 La Ley de Presupuestos Generales del Estado (PGE) para 2021 deja temporalmente en suspenso esta exigencia. La regla de oro en CC LL se basa en la disposición final trigésimo primera de la Ley 17/2012, de 27 de diciembre, de Presupuestos Generales del Estado para el año 2013 , que permitía a las CC LL y sus entes dependientes contratar nuevo endeudamiento a largo plazo para financiar inversiones bajo ciertas condiciones.
} 
las finanzas públicas. Sobre la base de estos tres criterios se discute a continuación.

\subsection{La calidad de las finanzas públicas}

El marco actual de las reglas fiscales ha contribuido a crear una cultura de disciplina fiscal y de buena gestión financiera pública en la mayoría de los GSN. La remisión de información, los elementos de transparencia, las publicaciones de la información financiera y la supervisión por parte de los órganos de tutela han dado lugar a una nueva cultura para los gestores. Esta dimensión se ha reforzado con foros de diálogo en los que la Autoridad Independiente de Responsabilidad Fiscal (AIReF) también está cada vez más presente y en los que se debate sobre las reglas fiscales $y$ los problemas que pueden presentar.

En estos foros hay que destacar dos, que tienen un origen normativo previo: el Consejo de Política Fiscal y Financiera (CPFF), con las CC AA, y la Comisión Nacional de la Administración Local (CNAL), con las CC LL. Otros se han creado posteriormente a iniciativa del Ministerio de Hacienda, como la reunión anual de los representantes de los Órganos de Tutela o el grupo de trabajo para la aplicación de la regla de gasto en las CC LL. Por lo tanto, se puede concluir que, desde esta perspectiva, el marco fiscal actual sí ha contribuido a mejorar la calidad de la gestión de las finanzas de las AA PP.

Sin embargo, desde otro punto de vista, algunos autores han cuestionado de manera complementaria la contribución de este marco normativo a mejoras cualitativas en la gobernanza fiscal de las CC AA, destacando otros factores que tienen importancia en el comportamiento fiscal de los GSN, tales como los institucionales y/o políticos. Es el caso de Delgado-Téllez et al. (2017), para quienes un marco con reglas fiscales estrictas como el español no ha supuesto un impacto significativo en la reducción de los incumplimientos. En una línea similar, Martínez López (2020b) ha descrito la reducida eficacia de la LOEPSF y su aplicación para cumplir los objetivos de déficit, facilitar una buena ejecución de la política fiscal durante el periodo transitorio y dotar de credibilidad a los instrumentos preventivos y correctivos, entre otros.

Para conseguir avances en esta dirección se requieren, no obstante, arreglos institucionales entre el Estado y los GSN que vayan más allá de los marcos de reglas fiscales y aseguren procedimientos de aplicación que sean políticamente creíbles. En este sentido, la literatura ha subrayado la importancia de la coordinación (Lago, 2017), de los factores reputacionales y de mimetismo (Molina Parra y Martínez López, 2018), de la factibilidad de los objetivos (Fernández Llera, 2016) e incluso de la propia consistencia intertemporal del Gobierno central (Martínez López, 2021). Aunque se reconoce que la reforma que supuso la LOEPSF tuvo su importancia en mejorar la gestión financiera de los GSN, existen dudas sobre si el marco actual ha respondido adecuadamente a incrementar la corresponsabilidad de los GSN, si existen brechas entre el diseño y la implementación del marco y si el estado actual de la descentralización fiscal es el adecuado para cumplir objetivos de consolidación fiscal.

Otro vector de discusión acerca del éxito relativo de la actual gobernanza fiscal exige tratar los importantes problemas relacionados con la reforma de los sistemas de financiación autonómica y local (Instituto de Estudios Fiscales, 2018). Una primera aproximación requeriría atender la posible existencia de desequilibrios fiscales verticales entre CC AA y CC LL y, en una segunda derivada, las inequidades $\triangleright$ 
horizontales -especialmente en el ámbito autonómico- que pueden condicionar sin duda los esfuerzos de consolidación fiscal o al menos la legitimidad para exigirlos (Dovis y Kirpalani, 2020; Martínez López, 2020a).

Como una extensión de lo anterior, habría que atender a los problemas de incentivos y de riesgo moral creados por sistemas de mutualización de deuda que suponen los mecanismos extraordinarios de financiación. Se requiere una profunda reconsideración de estos mecanismos para aproximarlos a su verdadera naturaleza de recurso de última instancia (Lago, 2017) al tiempo que mitigar los incentivos perversos al sobreendeudamiento (Cuenca, 2015).

\subsection{Propiedades anticíclicas de las reglas fiscales}

En este punto conviene atender principalmente a cómo se ha conjugado la evolución del ciclo económico con los esfuerzos de consolidación fiscal. Así, de un lado, podemos constatar que durante la vigencia de la LOEPSF se ha producido una notable reducción del déficit en el conjunto de las AA PP. No obstante, esta afirmación debe cualificarse en la medida en que desde 2014 la economía española experimenta tasas de crecimiento del PIB positivas (y desde 2015 hasta 2019 por encima del 2\%), con lo que la mayor parte del esfuerzo por reducir el déficit ha venido de factores coyunturales. Con otras palabras, el déficit estructural al final del periodo observado es tan elevado como al principio del mismo, poniendo de manifiesto que la orientación de la política fiscal no ha sido precisamente contractiva (Hernández de Cos, 2020).

En el Gráfico 1 se observa la evolución del conjunto de las AA PP, sin considerar todavía el enorme incremento del déficit público que se alcanzará en 2020 y 2021. Puede apreciarse cómo la reducción del déficit es generalizada $\triangleright$

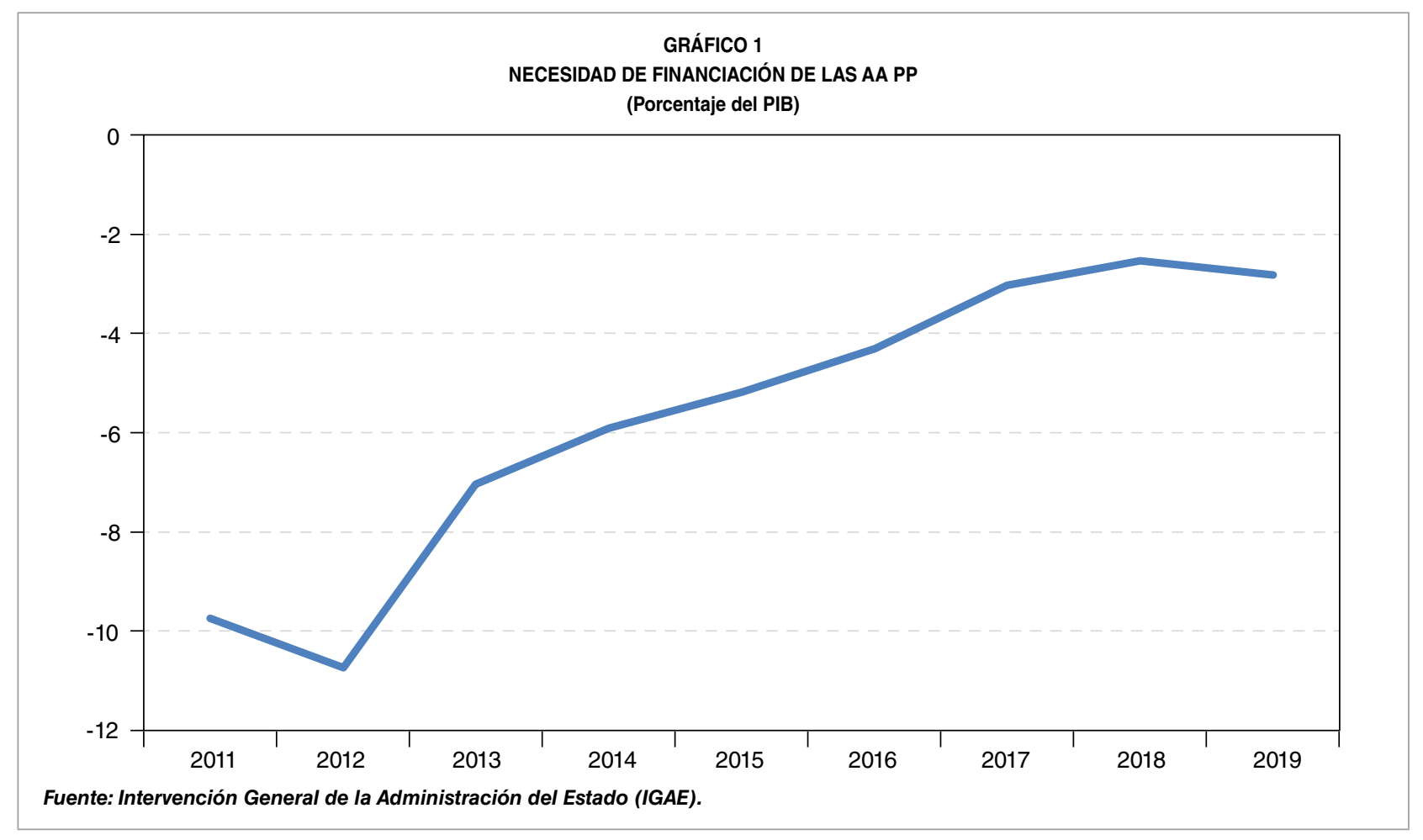


Fernando González y Diego Martínez López
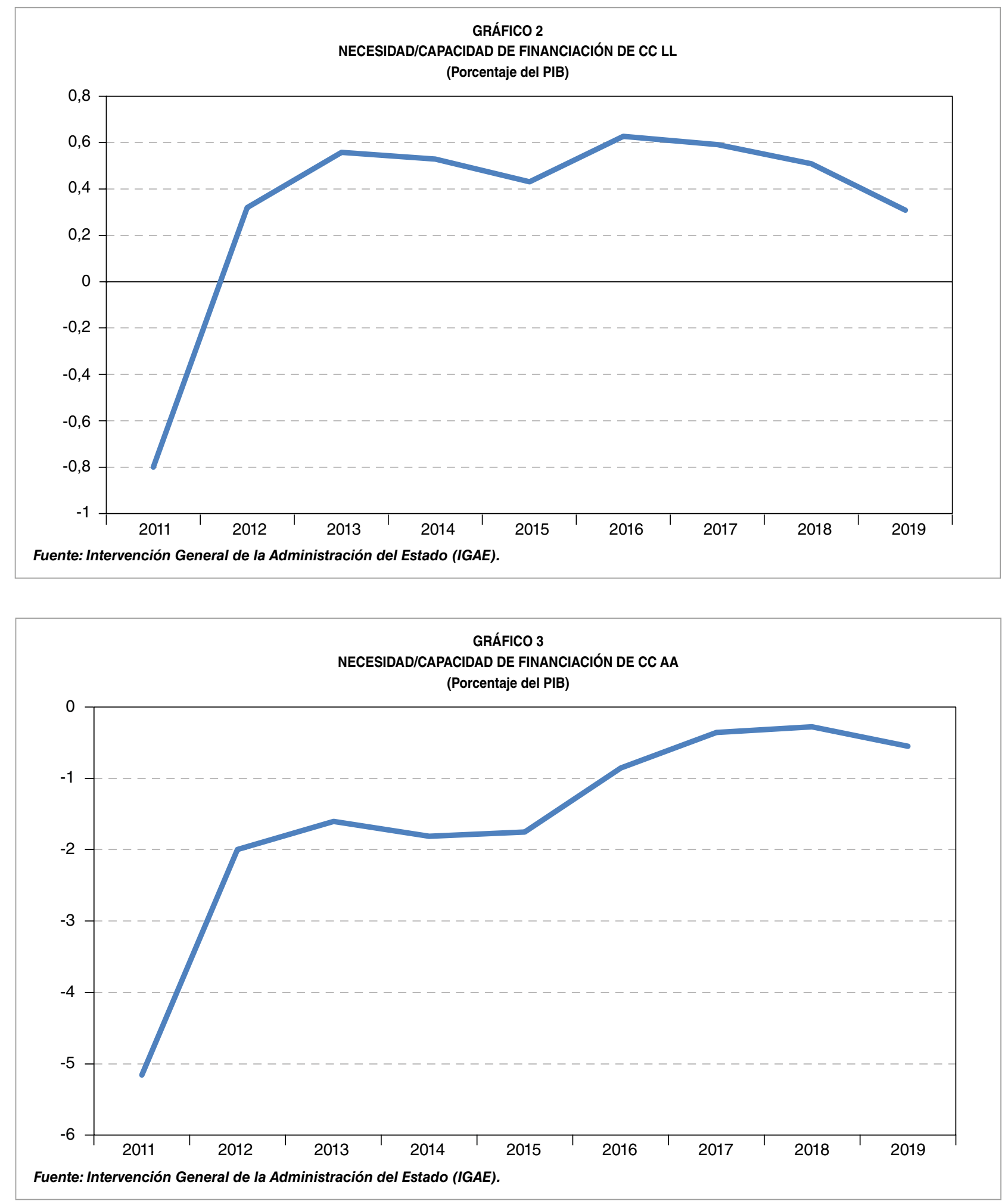

entre los GSN en España, con especial énfasis en las CC LL, si bien esta trayectoria descendente se ha visto frenada en los años 2015 y 2019, en que coincidieron procesos electorales.
Precisamente, en torno a las diferencias entre CC AA y CC LL cabe una discusión adicional en la medida en que sus resultados de déficit pueden estar condicionados por la $\square$ 
posible existencia de un desequilibrio vertical. Dicho de otro modo, cabe cuestionarse si los recurrentes superávits de las CC LL son fruto de su mayor disciplina fiscal o de una beneficiosa distribución de empleos y recursos (más inelásticos al ciclo en el ámbito local). Así, comparada esta distribución con la de las CC AA, se podrían obtener saldos excedentarios locales más allá de las necesarias dosis de rigor fiscal (Gráficos 2 y 3). Posiblemente los dos factores tengan algo que decir, y una investigación rigurosa al efecto sería bienvenida.

\subsection{La sostenibilidad a largo plazo de las finanzas públicas}

La vigencia de la normativa sobre disciplina fiscal ha coincidido con un importante incremento en la deuda pública de todas las AA PP, de manera que se ha llegado a niveles próximos al $100 \%$ del PIB antes incluso de la pandemia. Cuando los datos comiencen a reflejar el enorme impacto de la pandemia sobre las cuentas públicas, el nivel de endeudamiento público de España podría alcanzar el $120 \%$ a nivel país (Hernández de Cos, 2020).

En un primer momento, a principios de la década de 2010, gran parte del incremento en deuda pública fue el resultado de convertir deuda comercial de Administraciones territoriales en deuda financiera (Gráfico 4). En efecto, con los mecanismos extraordinarios de financiación la mayor parte de la deuda comercial de las CC AA y EE LL se fue saldando y se transformó en préstamos entre el Estado y las Administraciones territoriales (Ministerio de Hacienda, 2020a y 2020b). $D$

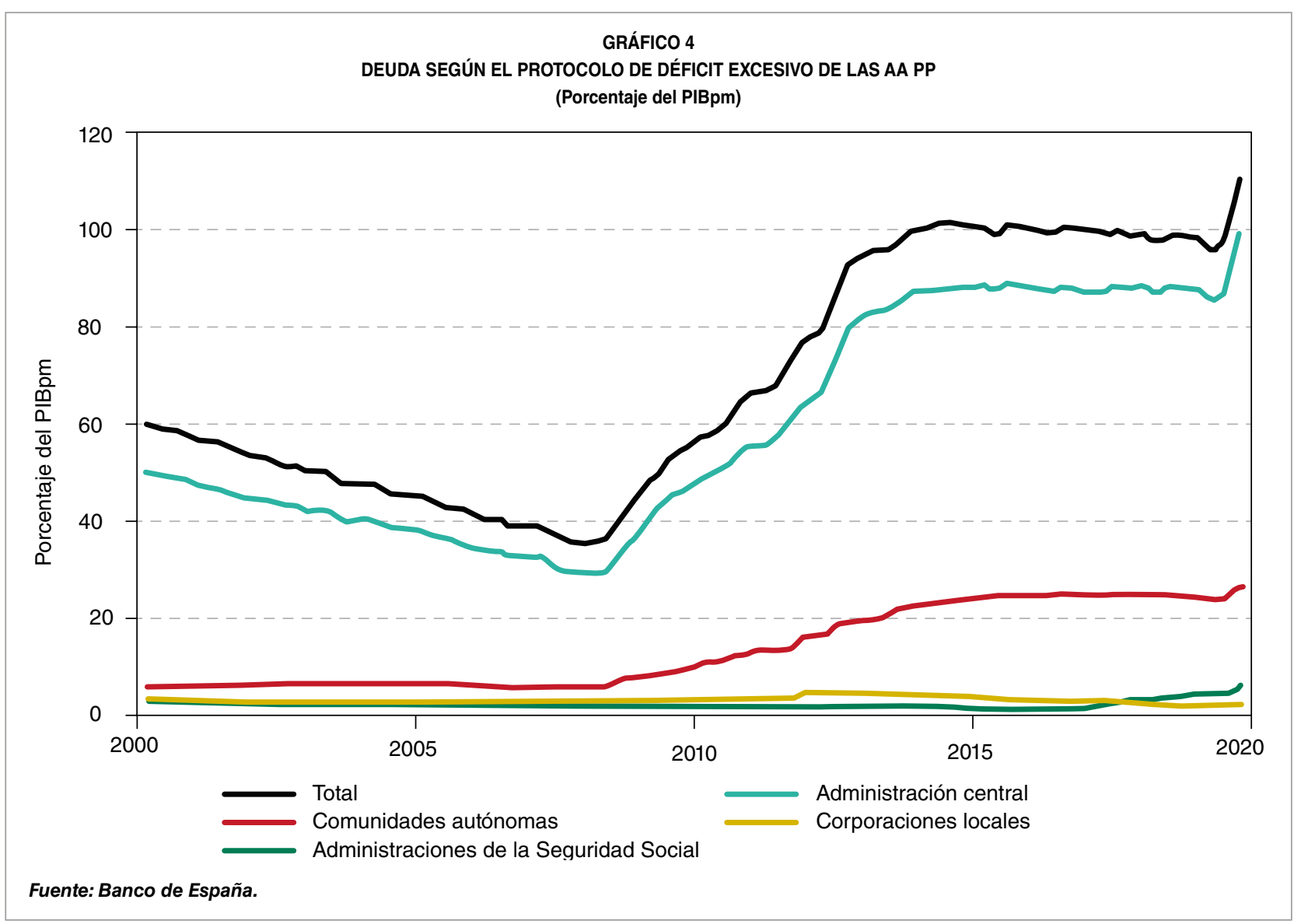


El Estado tuvo que acudir a los mercados financieros para poder otorgar estos préstamos a los GSN.

En estas condiciones, la gestión y reducción de este elevado volumen de deuda pública se constituye pues como el principal reto al que se enfrentan las finanzas públicas españolas a corto y medio plazo, y, como sugerimos posteriormente, debe ser la referencia que guíe el rumbo de las futuras reformas.

Por otra parte, no hay que perder de vista que, durante la consolidación fiscal, el gasto en inversión pública se ha resentido en todos los niveles de la Administración. Así, como demuestra el Gráfico 5, el porcentaje de gasto corriente en relación con el PIB se ha mantenido relativamente estable en torno a 14 y al 6 en CC AA y CC LL, respectivamente. Por su parte, la proporción del gasto de inversión se ha reducido hasta valores en torno al 1 y al $0,5 \%$, tanto en CC AA como en CC LL, respectivamente (Gráfico 6).
Este sesgo favorable hacia el gasto público corriente y en detrimento del de inversión supone una obvia limitación para el potencial de crecimiento futuro. Si bien es cierto que los factores determinantes de esta circunstancia pueden ser muy variados, desde la estructura demográfica por edades hasta elementos de economía política (Delgado-Téllez y otros, 2020), al igual que en el marco europeo conviene una reflexión sobre qué responsabilidad tienen las actuales reglas fiscales en esta incapacidad para garantizar una protección mínima a la inversión pública.

Llegados a este punto, podemos concluir que el marco actual de reglas fiscales ha contribuido a la consolidación fiscal y a la creación de una cultura de gestión financiera pública superior a la anterior. Pero también hay que reconocer que no ha sido lo suficientemente eficaz para evitar la acumulación de deuda en las AA PP ni ha protegido el gasto en inversión, amén de otros problemas de coordinación y gobernanza.

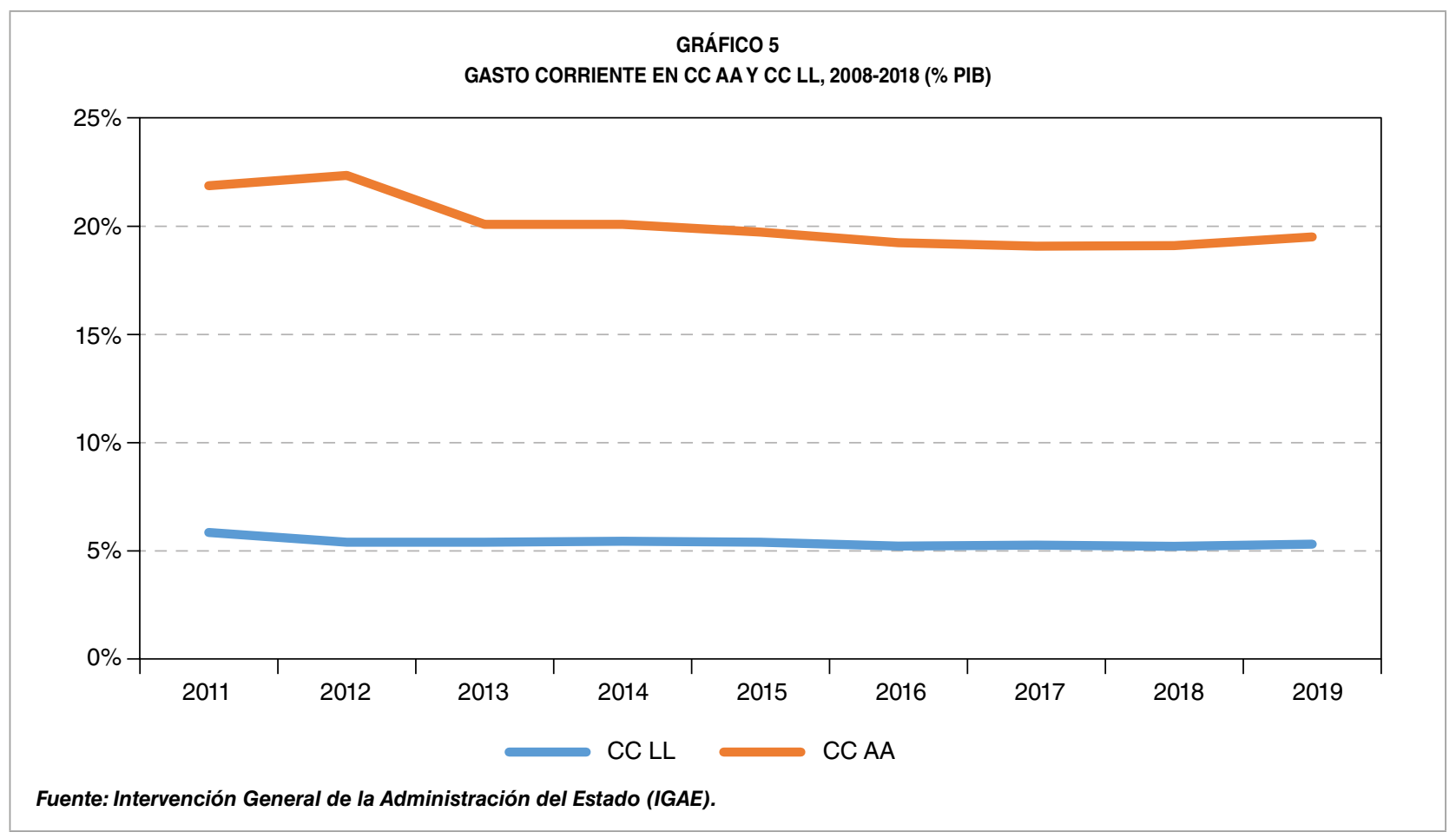




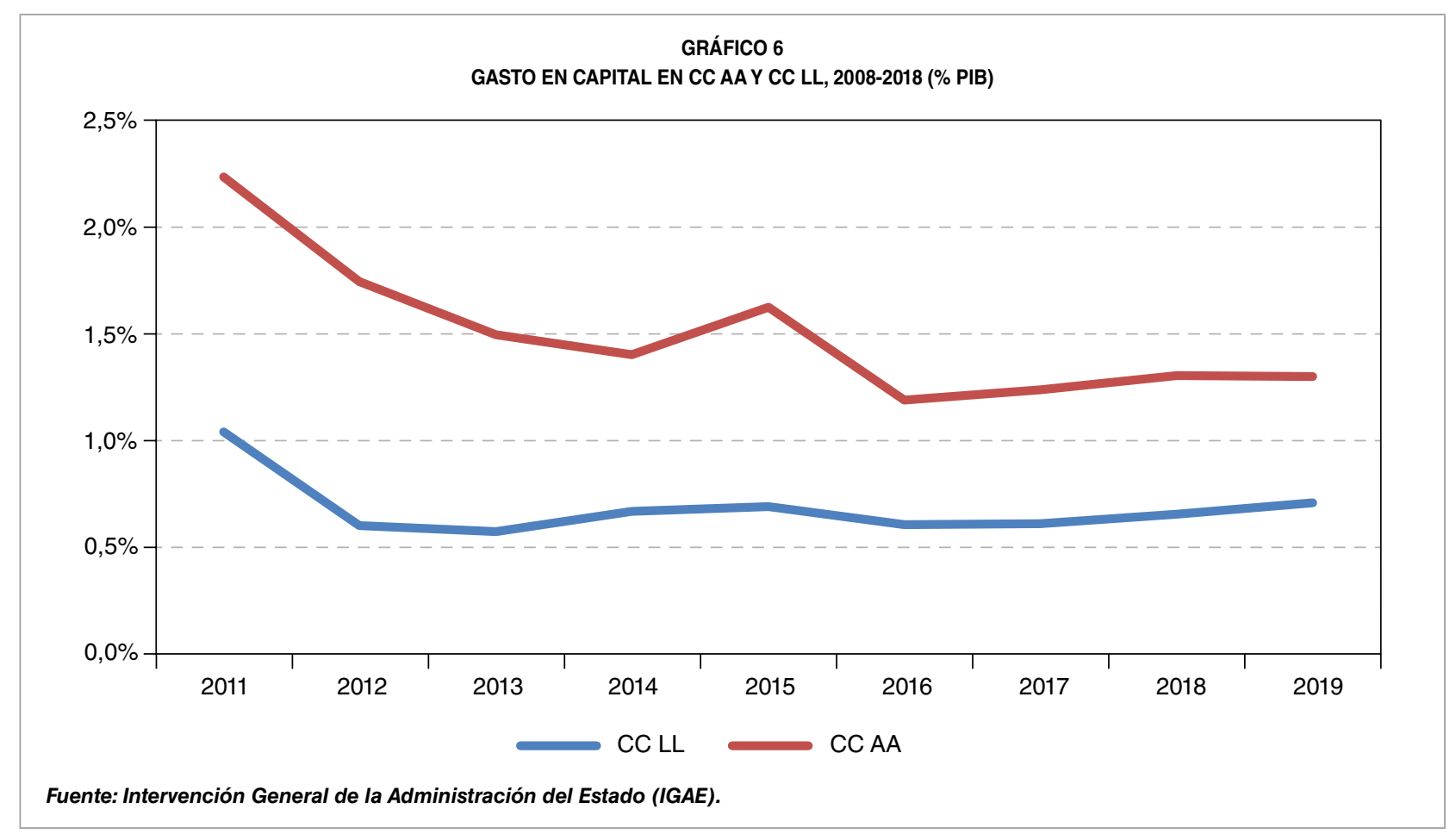

\section{Problemas de diseño}

Esta sección identificará determinados problemas de diseño del actual marco de gobernanza fiscal en lo relativo a los GSN en España. Para ello seguiremos ciertos criterios que la literatura ha identificado como deseables en el caso específico del diseño de reglas fiscales aplicables a GSN (Eyraud et al., 2018, 2020).

\subsection{La coherencia entre las reglas}

La LOEPSF no ha establecido ninguna metodología concreta para la fijación de objetivos. Sí que recomienda una coherencia entre ellos. Por ejemplo, se establece que para la fijación del objetivo de estabilidad presupuestaria se tenga en cuenta la regla de gasto y el saldo estructural alcanzado en el ejercicio inmediato anterior. También que la fijación del objetivo de deuda pública sea coherente con el objetivo de estabilidad presupuestaria establecido.

Igualmente se apunta que, si en los supuestos de aplicación de cláusulas de escape se superan los límites de endeudamiento, el objetivo deberá garantizar una senda de reducción de la deuda pública acorde con la normativa europea. Por otra parte, la propuesta de fijación de los objetivos de estabilidad presupuestaria y de deuda pública debe cumplir con las recomendaciones y opiniones emitidas por las instituciones de la Unión Europea. Pero más allá de estos condicionantes, no existe en la LOEPSF ni en su normativa de desarrollo ninguna metodología para el cálculo de los objetivos.

Ello hace que se produzcan determinadas incoherencias entre las reglas. En primer lugar, no existe ninguna conexión entre la tasa de referencia aplicable a la regla de gasto y la posición fiscal, ni entre la primera y el objetivo de déficit. Además, el establecimiento de $\triangleright$ 
objetivos de déficit idénticos a todas las CC AA no favorece precisamente la necesaria coherencia entre las reglas fiscales ${ }^{3}$.

En segundo lugar, tampoco existe la obligada conexión entre el objetivo de déficit y el de deuda, en el sentido de establecer primero el de deuda a fin de converger a un nivel deseable de endeudamiento. En la actualidad, más bien ocurre lo contrario: se fija primero el de déficit $y$, partiendo del stock de deuda existente y sumando el objetivo de déficit, se fija el de deuda. De este modo, si se cierra finalmente con un déficit inferior al objetivo (o incluso superávit), la diferencia supone una sobrefinanciación de las necesidades de endeudamiento. En rigor, el cierre de un ejercicio en términos de déficit debería tenerse en cuenta para la fijación del objetivo de deuda del año siguiente. Y esta no ha sido la práctica habitual hasta muy recientemente.

Y, en tercer lugar, como ha señalado la AIReF (2018), y en línea con algunas propuestas a nivel europeo (European Fiscal Board, 2019), puede demostrarse que la formulación actual de la regla de gasto no permite un acercamiento hacia el objetivo de medio plazo (en términos de deuda pública). En el mejor de los casos, el cumplimiento de la actual regla de gasto conduce a una orientación neutral de la política fiscal, insuficiente para reducir la deuda de manera sustancial y dados los elevados déficits estructurales que se vienen experimentando.

\subsection{Transparencia y sencillez}

La inconsistencia entre las reglas fiscales anteriormente expuesta ha creado, entre otros

3 En el caso de las CC LL no procede, por obvias razones operativas, la discusión acerca de objetivos de déficit individuales. motivos, un marco regulatorio difícil de manejar y aplicar en su completitud. Como resultado, la vigilancia y la aplicación de las reglas es un ejercicio que resulta demasiado complejo y poco transparente. La complejidad es un rasgo común de las denominadas reglas fiscales de segunda generación al que no escapa nuestro sistema (Eyraud et al., 2018).

Estos nuevos marcos de reglas fiscales surgidos en la década pasada se caracterizan por ser más flexibles en su diseño en relación con las condiciones macroeconómicas pero más restrictivos en la gestión del presupuesto, incluyendo mecanismos de vigilancia y aplicación mejorados. Esta mayor flexibilidad ha venido a costa de añadir complejidad y, a la larga, se han generado sistemas de reglas difíciles de seguir y de entender por los ciudadanos, con lo que se complica la economía política de los procesos de consolidación fiscal. Partiendo del trade-off entre flexibilidad y simplicidad, sería deseable reducir el nivel actual de complejidad del marco de reglas fiscales para incrementar su transparencia (European Fiscal Board, 2019; Comisión Europea, 2020).

Un ejemplo ilustrativo del precio pagado en forma de menor transparencia y sencillez, en aras de una mayor flexibilidad, puede encontrarse en la importancia que el concepto de déficit estructural tiene en las actuales normativas de gobernanza fiscal. Como es sabido, se trata de una magnitud no directamente observable, y eso ya impone un primer obstáculo a la necesaria claridad exigible a las reglas fiscales. De forma adicional, la estimación del saldo estructural no está exenta de complicaciones metodológicas y de calendario.

Y en el caso de los GSN, todas estas circunstancias multiplican su impacto. Un posible enfoque para asignar una brecha de producto para Gobiernos subnacionales es utilizar $\triangleright$ 
una asignación desde arriba hacia abajo, partiendo de una brecha global a nivel país, que se va distribuyendo entre las Administraciones. La brecha de producto a nivel nacional se divide primero entre el componente del Gobierno central y el componente subnacional, dividiendo el parámetro de semielasticidad presupuestaria (que estima la sensibilidad del saldo presupuestario a la brecha de producto) en dos valores, uno para el Gobierno central y otro para los GSN considerados en conjunto. La segunda etapa consiste en dividir ese componente subnacional entre GSN, lo cual puede hacerse en función del tamaño relativo de sus ingresos. Para este segundo paso, la normativa española ha optado por un supuesto excesivamente simplificador, que implica que «se utilizarán las mismas elasticidades y brecha de producción que a nivel nacional ${ }^{4}$. $Y$ ello a pesar de que existe evidencia sobre la heterogeneidad de los ciclos económicos regionales (Gadea et al., 2012).

Por supuesto, a escala local ni siquiera merece la pena considerar la aplicación de conceptos como el de déficit estructural. Pero incluso en el ámbito regional la estimación de saldos presupuestarios ajustados cíclicamente exige la utilización de supuestos simplificadores de dudoso encaje con la realidad.

\section{Flexibilidad de las reglas en respuesta al ciclo}

Las reglas fiscales son «flexibles» cuando sus límites numéricos aplicados a los agregados fiscales pueden ser ajustados según el ciclo o suspendidos temporalmente bajo

4 Véase la Orden ECC/1556/2016, de 28 de septiembre, sobre el cálculo de las previsiones tendenciales de ingresos y gastos y de la tasa de referencia de la economía española. circunstancias especiales. Por consiguiente, la necesidad de ajustar los límites numéricos de las reglas fiscales ocurre normalmente en dos contextos: la estabilización económica y la respuesta a eventos imprevistos.

En el caso de la estabilización económica, las reglas fiscales flexibles se utilizan para permitir límites suficientemente amplios de déficit en tiempos de recesión, a fin de que actúen los estabilizadores automáticos, y, por el contrario, para fijarlos de forma más restrictiva en tiempos de expansión. En el caso español, además, se da la circunstancia de que existen estabilizadores automáticos relativamente potentes a nivel subnacional, dado que la gran mayoría de sus ingresos autonómicos (en torno al $80 \%$ ) viene determinada por sus ingresos en el sistema de financiación autonómica, que a su vez dependen en gran parte de su participación en la recaudación de impuestos. En las CC LL, por el contrario, las bases imponibles de los impuestos locales son más inelásticas al ciclo.

Existen varias formas de flexibilidad en las reglas fiscales: las ya citadas reglas fiscales ajustadas por el ciclo, las reglas de gasto al uso y las reglas de equilibrio presupuestario combinadas con fondos de acumulación. En nuestro país hemos utilizado las dos primeras. En cuanto a las reglas de gasto, estas suelen suponer una solución conceptualmente senciIla para que la política fiscal resulte contracíclica. Ciertos inconvenientes de diseño afectan, sin embargo, a la configuración actual de la regla de gasto en España. En el contexto de GSN, esta suele presentar problemas fundamentalmente derivados de la rigidez del gasto (corriente) que tienen encomendado y la consiguiente utilización de la inversión pública como variable de ajuste.

Yendo a un plano más concreto, la configuración actual de la regla de gasto ha dado $\triangleright$ 
lugar a importantes controversias en el caso de GSN, especialmente en cuanto a su aplicación en un contexto dinámico. En particular, su medición a partir de presupuestos liquidados, sin ningún tipo de ajuste en ejercicios siguientes en función del grado de cumplimiento, genera importantes problemas de consolidación del gasto (en ambos sentidos, tanto si se ha cumplido como si no).

Con otras palabras, un incumplimiento muy elevado al principio permite «vivir del incumplimiento", dado que no existe una corrección de la tasa de crecimiento máximo al año siguiente. De manera similar, si se ha cumplido y se ha tenido un gasto por debajo del máximo determinado por la regla, esta interpretación restringe la capacidad en años sucesivos, generando incentivos a incumplir o a quedarse lo más cerca del límite posible. La AIReF se ha pronunciado en repetidas ocasiones en contra de esta forma de medir el cumplimiento de la regla de gasto (véase, por ejemplo, AIReF, 2017). Esta interpretación de la regla de gasto también afecta al diseño y verificación de los planes económico-financieros.

Dados estos problemas para conjugar estabilización económica y sostenibilidad en las reglas fiscales, una tercera aproximación para otorgar flexibilidad al marco de reglas fiscales es la que establece reglas de equilibrio presupuestario combinadas con fondos de acumulación (Eyraud et al., 2020). Estos autores han recomendado la combinación entre una regla de equilibrio nominal y un fondo de acumulación (rainy day fund, RDF). Este último implica la obligación de reservar ahorro cuando la posición fiscal está saneada, de manera que pueda utilizarse cuando la situación fiscal empeore. Aunque se trata de una idea atractiva desde el punto de vista conceptual y utilizada con eficacia en otros ámbitos de la Administración (véase el fondo de reserva de la Seguridad Social), la ausencia de referentes internacionales en países comparables con España que pudieran servir de guía y la difícil economía política que se intuye para gestionar su uso (¿cuándo y cuánto aportar?, ¿en qué condiciones se pueden retirar fondos?) hacen complicado a nuestro juicio su utilización en un contexto como el español.

La flexibilidad de las reglas fiscales también puede abordarse a través de las cláusulas de escape que permiten excepcionar el cumplimiento fiscal. Se trataría de responder a eventos inesperados como desastres naturales, conflictos y recesiones económicas severas, que reduzcan de forma importante los ingresos y que requieran incurrir en gastos importantes. El impacto de la COVID-19 es un reciente ejemplo, en tiempo presente, de este tipo de eventos.

En la normativa española se establece la posibilidad de que, excepcionalmente, el Estado y las CC AA (no las CC LL) puedan incurrir en déficits estructurales en caso de catástrofes naturales, recesión económica grave o situaciones de emergencia extraordinaria, al tiempo que se permite superar los límites de deuda pública establecidos. Estas circunstancias excepcionales deben ser apreciadas por la mayoría absoluta de los miembros del Congreso de los Diputados. Asimismo, se requiere que esta desviación temporal no ponga en peligro la sostenibilidad fiscal a medio plazo.

La pandemia de la COVID-19 es un caso claro de una situación que permitiría la activación de la cláusula de escape. De hecho, desde diversas fuentes se ha venido pidiendo su activación (Martínez López, 2020a; AIReF, 2020), que finalmente ha culminado en octubre de 2020 con la apreciación por parte del Congreso de los Diputados de $\triangleright$ 
que dichas circunstancias excepcionales se han producido ${ }^{5}$.

\section{Protección del gasto de inversión}

Un aspecto importante a considerar a la hora de diseñar un marco de reglas fiscales para GSN es la necesidad de dejar un espacio suficiente a la realización del gasto en inversión pública. En torno a la mitad del gasto de inversión de las AA PP en los países de renta alta es realizado por los GSN (OECD, 2016). En este sentido, algunas reglas fiscales son más restrictivas que otras, y el diseño debería siempre tener en consideración la protección de este tipo de gasto, con efectos beneficiosos para las economías en el largo plazo (ver discusión en Eyraud et al., 2020). Entre las más utilizadas se encuentran las reglas de equilibrio en términos corrientes, las reglas de oro y techos de gasto corriente. En el caso español, existen reglas de oro aplicables a los GSN, aunque en el caso de las CC AA temporalmente desactivadas para 2021. Por otra parte, con especial encaje en las CC LL, se han adoptado medidas de protección del gasto de inversión pública a través del tratamiento de las inversiones financieramente sostenibles (IFS).

Estas IFS se definen como aquellas inversiones que en el largo plazo tienen un impacto positivo, directo y verificable sobre la sostenibilidad de las finanzas públicas y el crecimiento potencial de la economía. El importe del gasto realizado en inversiones financieramente sostenibles no se considera como gasto computable a efectos de la aplicación de la regla de gasto, aunque sí a efectos del cumplimiento del

5 Para una valoración crítica de cómo se ha activado la cláusula de escape, véase por ejemplo Jimeno y Martínez López (2020). objetivo de estabilidad presupuestaria. Las IFS son, además, una excepción a la regla recogida en la LOEPSF de destinar el importe del superávit a reducir el endeudamiento.

La utilización de las IFS ha sido variable según los subsectores, con amplia incidencia en las CC LL pero muy reducida en las CC AA. En cualquier caso, la cuantía de las IFS ha mostrado una notable sensibilidad con respecto a la magnitud del superávit y, en cierta medida, a la inestabilidad jurídica que conllevaba que cada año se prorrogase en la correspondiente ley de PGE que, como es sabido, no ha tenido una secuencia normalizada.

En ausencia de un planteamiento general, la estrategia seguida por el legislador español ha sido la de ir cediendo progresivamente a la presión de los decisores públicos locales para ampliar el concepto de IFS, incluyendo cada vez nuevos programas de gasto que permitieran superar la rigidez de la normativa actual. Sin embargo, este tipo de soluciones no resultan satisfactorias en términos integrales $\mathrm{y}$, además, pueden dar lugar a problemas de incentivos por una incorrecta contabilización y clasificación del gasto para encajarlo dentro del concepto de IFS ${ }^{6}$.

Tampoco las reglas de oro son, en nuestra opinión, la mejor fórmula para proteger el gasto en inversión pública. Dada la elevada laxitud de la política monetaria, con tipos de interés negativos para la deuda soberana, y ante la situación generada por la pandemia, no parece razonable imponer restricciones adicionales al endeudamiento, más allá de la prudencia financiera y obviamente -aunque no es superfluo recordarlo- la aplicación de estos recursos $\triangleright$

\footnotetext{
6 Tanto a nivel europeo como en la primera reforma que experimentó la normativa española sobre estabilidad en 2006 ya se atisbaba el riesgo de "camuflar" gasto corriente como de capital para recibir un mejor tratamiento a efectos de disciplina fiscal (Martínez López, 2007)
} 
a proyectos de elevada productividad y no a elefantes blancos (Blanchard et al., 2020).

Una posibilidad para mejorar el marco actual sería considerar el gasto público en inversión con un horizonte temporal mayor al anual de la regla de gasto. Se trataría de adoptar un esquema similar al de la regla de gasto de la UE, en la que el gasto computable se mide «suavizando» el componente de la inversión a través del cálculo promedio de cuatro años, para evitar el impacto que puedan tener en años puntuales los proyectos grandes de inversión. Otra solución, que apuntaremos en la sección final, sería modular este tipo de gasto según el nivel de endeudamiento dentro de la regla de gasto.

\section{Acceso al endeudamiento}

En este apartado analizaremos varios aspectos relacionados con el acceso al endeudamiento de los GSN. Como apuntábamos anteriormente, si bien el marco actual ha podido contribuir en años anteriores a una cierta consolidación fiscal (apenas perceptible en términos estructurales), lo que resulta evidente es que ha generado un alto nivel de endeudamiento en las haciendas subcentrales en general y en las CC AA en particular. La gestión y reducción de este endeudamiento público será el principal problema al que se enfrentará la política fiscal de los próximos años.

\section{Los mecanismos extraordinarios de}

financiación: el Fondo de Financiación de Comunidades Autónomas (FFCCAA) y el Fondo de Financiación de Entidades Locales (FFEELL)

La propia razón de ser de estos mecanismos es una cuestión disputada en la literatura.
De un lado, está admitido que el recurso al endeudamiento de los GSN puede tener como objeto la estabilización económica. Sin embargo, desde el punto de vista de la teoría del federalismo fiscal, la función de estabilización económica no debería estar asignada a GSN. Ello es debido a que las políticas contracíclicas centralizadas tienen ventajas en casos de shocks tanto asimétricos como simétricos. Además, los Gobiernos centrales generalmente pueden endeudarse a un coste menor que los GSN, disponen de bases tributarias mayores y un flujo de ingresos más estable a lo largo del ciclo.

De otro lado, sin embargo, extensiones adicionales del federalismo fiscal establecen que los GSN deberían financiarse no solamente con recursos propios autogenerados y transferencias desde el Estado, sino que una cierta parte de sus necesidades de financiación puede provenir del recurso al endeudamiento. Ello sería especialmente adecuado en el caso del gasto en inversión pública, con efectos acotados desde el punto de vista espacial, e intentando que la corriente de beneficios futuros se correspondiese con las amortizaciones de préstamos (Eyraud et al., 2020).

En este sentido, el esquema español representado por los mecanismos extraordinarios de financiación se correspondería con el primero de los argumentos: la política de estabilización recae fundamentalmente en el Estado y este comparte los ahorros de su mejor acceso a la financiación con las Administraciones territoriales, hasta el punto de ofrecer incluso financiación no reembolsable, como es el caso del Fondo COVID-19.

En su origen, de hecho, la financiación extraordinaria venía motivada por la necesidad de otorgar liquidez a los GSN, que vieron cerrado su acceso a los mercados financieros $D$ 
con la crisis iniciada en 2008-2009, al tiempo que se intentaba reducir su deuda comercial. Todo ello acompañado de la correspondiente condicionalidad fiscal. En este sentido, la finalidad de los préstamos no se ajusta al objetivo del endeudamiento de GSN para fines de inversión que prescribe otra parte de la literatura. Más arriba, de hecho, hemos comprobado cómo no han contribuido a proteger el gasto público en inversión frente al corriente.

Sin embargo, en el momento actual, los GSN tienen acceso a unos mercados de capitales que, por otra parte, cuentan con suficiente liquidez como para absorber volúmenes importantes de deuda en condiciones favorables. En este contexto, la financiación a través del Estado está menos justificada que en 2012. Y en otro orden de cosas, estos mecanismos han podido contribuir a los elevados niveles de endeudamiento actuales, como ya indicamos antes. Esto se debe a que detrás de los mecanismos extraordinarios de financiación subyace un problema de riesgo moral?.

En estas condiciones, la reforma de estos mecanismos debería encaminarse hacia una transición en la que se reconfiguren como un último recurso sujeto a fuerte condicionalidad fiscal, para casos de episodios de falta de liquidez inesperados, y no como un canal sistemático de financiación privilegiado frente al mercado.

\section{Sobrefinanciación de los déficits}

Una dimensión adicional del sobreendeudamiento en que han incurrido las CC AA tiene su origen no tanto en el diseño específico de la LOEPSF, sino más bien en su peculiar aplicación. En línea con lo apuntado anteriormente,

\footnotetext{
7 No obstante, un reciente trabajo de Calvo y Cadaval, (2021) encuentra que las CC AA que más se han beneficiado de estos fondos no han tenido un comportamiento fiscal más imprudente.
}

el método de fijación del objetivo de deuda individual que se ha utilizado tradicionalmente se basa en un objetivo de déficit no diferenciado que se añade al stock de deuda existente. Esta operativa puede suponer un mayor margen de endeudamiento que el estrictamente necesario para cubrir el déficit real.

\section{El destino de los superávits}

La aplicación de la LOEPSF en este tema es un ejemplo ilustrativo de cómo una previsión legal diseñada en su momento no previó circunstancias que se hicieron realidad al poco tiempo de ser aprobada la ley, a saber, la aparición de superávits en las Administraciones territoriales, en especial en las CC LL. Tampoco tuvo lugar un desarrollo normativo posterior que interpretase cómo debe realizarse esa reducción de endeudamiento, y todo ha quedado constreñido a liberar presión a través de las IFS y sus sucesivas ampliaciones.

Y también han surgido dudas interpretativas sobre la aplicación de la ley en este terreno. Por ejemplo, en el caso de que existan costes de amortización anticipada que hagan que la reducción del endeudamiento resulte costosa, se podría plantear si es compatible aplicar una parte del superávit a reducir ese endeudamiento y el resto a lo largo de varios años. También han surgido dudas en el caso de que el superávit sea recalculado a consecuencia de alguna reevaluación en términos de contabilidad nacional, con efectos retroactivos e imputables a varios años anteriores (por ejemplo, con la actualización del cupo vasco que tuvo lugar en 2017).

En todos estos casos es importante analizar el impacto del superávit en términos del criterio de caja. La AIReF ha reconocido que las ideas y propuestas relativas a la aplicación de la $D$ 
LOEPSF plantean problemas de encaje en la normativa vigente e insta a establecer un procedimiento para la aplicación de dicho artículo a las Administraciones territoriales. Para ello recomienda que se establezca un calendario de amortización que tenga en consideración las disponibilidades líquidas y los costes financieros asociados a la amortización anticipada de la deuda.

Regreso a la regla de oro

Respecto a la regla de oro, aunque está vinculada a la protección del gasto de inversión y parece prudente desde el punto de vista de la lógica financiera, es importante tener en cuenta que puede ser una regla fiscal muy restrictiva en determinadas situaciones, aparte de generar posibles incentivos a contabilizar gasto corriente como gasto de inversión, como ya hemos apuntado. Las reglas de oro fueron muy populares en su momento, pero diversas razones hacen que actualmente, en nuestra opinión, no sean aconsejables en la gobernanza fiscal española.

En primer lugar, el desarrollo de los mercados financieros actuales no es el mismo que en el momento en que se propusieron las reglas de oro. La oferta financiera actual es mucho más profunda y permite una financiación de las AA PP más acorde a sus preferencias intertemporales. Además, las preferencias sociales pueden haber cambiado en los últimos tiempos con el envejecimiento poblacional, sesgándose hacia el consumo actual frente al consumo futuro, por lo que no sería una regla con suficiente legitimidad intergeneracional. Por último, la situación actual de la pandemia puede hacer necesario recurrir al endeudamiento a largo plazo también para financiar gasto corriente de emergencia. Por ello, aunque la protección del gasto de inversión es deseable como objetivo de las reglas fiscales, en nuestra opinión no es a través de las reglas de oro la mejor manera de conseguir este fin. Así, en una situación como la actual, la regla de oro no se configura a nuestro juicio como la solución óptima para garantizar un gasto en inversión pública aceptable.

\section{Dos propuestas a futuro}

Un nuevo marco de reglas fiscales debe partir del reconocimiento de la situación actual: un gasto inesperado asociado a la crisis de la COVID-19 que derivará en un elevado nivel de déficit primero y un marcado incremento del endeudamiento de las AA PP después, que, a excepción de la local, ya estaban en niveles muy elevados.

En este contexto, está claro que en el corto plazo es necesario actuar a través de una política fiscal expansiva y medidas de liquidez que contengan los efectos negativos de la recesión, como se ha venido haciendo en meses anteriores. Por ello, es importante que el marco de reglas sea lo suficientemente flexible como para permitir el crecimiento de esos niveles de déficit y deuda en el corto plazo. Pero que también tienda a ir reduciendo el endeudamiento en el medio y largo plazo, una vez que la economía recupere el crecimiento.

Aparte de tener en cuenta la nueva realidad tras la pandemia, una reforma del sistema español de reglas fiscales debería mejorar el marco actual en términos de simplicidad y transparencia, garantizando una coherencia entre los objetivos y solucionando los importantes problemas institucionales y de incentivos que se han generado en el marco actual. En este sentido, habría que dotarlo de más automatismo y hacerlo menos dependiente de $D$ 
las sanciones, con una apuesta decidida por la corresponsabilidad de los GSN, en coherencia con el alto grado de descentralización del gasto alcanzado actualmente.

Además, también deberían abordarse los problemas relativos a la financiación de los GSN, con reformas pendientes en los sistemas de financiación autonómica y local, los relativos a los mecanismos extraordinarios de financiación, determinadas inconsistencias en el calendario presupuestario que dificultan la presupuestación de los GSN por circunstancias meramente políticas asociadas a procesos electorales, y otras relativas a los órganos de coordinación entre el Estado y los GSN tales como el Consejo de Política Fiscal y Financiera y la Comisión Nacional de la Administración Local. Finalmente, también como prerrequisito para una reforma de la gobernanza fiscal española, merecería la pena considerar si no sería más apropiado contar con reglas diferenciadas para los dos subsectores.

Sin embargo, estos últimos aspectos, que podríamos considerar prerrequisitos y relacionados con factores institucionales y de aplicación, siendo fundamentales para el buen funcionamiento de un futuro sistema de reglas fiscales reformado, no son objeto de este trabajo. Sí expondremos, por el contrario, de manera sucinta, dos propuestas alternativas para el diseño de las reglas fiscales de los GSN de nuestro país. Ambas se encuentran alineadas con la actual y recientemente inaugurada discusión de la gobernanza fiscal europea (Comisión Europea, 2020).

La primera de esas propuestas, partiendo del realismo que supone la dificultad de reformar el marco de manera radical, intenta solucionar los problemas mencionados a lo largo de este trabajo, especialmente el de la coherencia entre los objetivos, a partir de un desarrollo metodológico de determinación de los objetivos de déficit. La segunda, más radical, se centraría en crear un marco simple y transparente basado en una sola regla de gasto mejorada. Ambas propuestas están inspiradas en la necesidad de reorientar el sistema de reglas fiscales al control de la deuda, que debe ser, a nuestro juicio, el principal objetivo que guíe la política fiscal en el medio y largo plazo.

\section{Propuesta 1. Determinación de objetivos de} déficit basada en la reducción de la deuda

Esta propuesta establece una metodología para la fijación de los objetivos de déficit del subsector CC AA y de cada una de ellas que sea coherente con una determinada reducción de la deuda a un ritmo predeterminado. En este sentido, sería necesario calibrar nuevos objetivos de deuda pública según los niveles actuales pos-COVID-19. Esta regla se aplicaría fundamentalmente a CC AA, dada la dificultad operativa de trasladar esta lógica a todas las CC LL. Pero en el caso de que se determinara para alguna corporación local un nivel de endeudamiento objetivo, también se podría aplicar la misma lógica que con las CC AA.

Nuestra propuesta toma como referencia el marco de la gobernanza fiscal europea en lo que a la deuda pública se refiere, que presentamos a continuación de manera muy estilizada. Sin perjuicio del detalle técnico que se requiera, puede establecerse que la regla que inspira la dinámica de la deuda dentro del marco fiscal europeo viene dada por la siguiente expresión (Hauptmeier y Kamps, 2020):

$$
a\left(d_{t-1}-d^{\star}\right)=\Delta \operatorname{capb}_{t}+\operatorname{capb}_{t-1}+\mu \operatorname{og}_{t}-\frac{i_{t}-y_{t}}{1+y_{t}} d_{t-1}
$$

donde $a$ es la velocidad de ajuste al nivel de deuda objetivo $\left(d^{\star}\right)$ desde el existente en el $\triangleright$ 
periodo anterior $\left(d_{t-1}\right)$, ambos en porcentaje del PIB; capb es el superávit primario cíclicamente ajustado; $\mu$ es la sensibilidad cíclica del saldo presupuestario; og $_{t}$ es el output gap; $i_{t}$ es el tipo de interés (implícito) de la deuda pública e $y_{t}$ es la tasa de crecimiento del PIB nominal potencial. Así, el valor de $a$ en la normativa europea se establece en 0,05 , esto es, el porcentaje anual al que debe reducirse el exceso de deuda pública sobre el valor de referencia del $60 \%$ fijado en el Tratado de Funcionamiento de la Unión Europea. Por su parte, el término $\mu \mathrm{og}_{t}$ es el déficit cíclico.

El marco institucional europeo presenta dos notables debilidades. Una está relacionada con su carácter procíclico, que puede advertirse fácilmente con un simple análisis de la expresión [1]. En efecto, ante un deterioro del saldo cíclico $\mu \mathrm{og}_{t}$ por desaceleración o recesión económica, la variación del superávit primario debería ser positiva, esto es, una orientación contractiva de la política fiscal, y viceversa. Una segunda limitación se sitúa en la notable complejidad técnica que acompaña el diseño y aplicación de las reglas fiscales europeas. A ello contribuye la siempre complicada estimación del déficit estructural o cíclicamente ajustado.

Nuestra propuesta decide abordar estas debilidades del siguiente modo. De un lado, ignora los efectos del ciclo económico, que sí se considera en la evaluación ex post de su cumplimiento. De otro lado, y en parte como consecuencia de lo anterior, simplifica considerablemente la metodología. Por consiguiente, reformulamos la expresión [1] suponiendo que no existe output gap:

$$
p b^{*}=\frac{i_{t}-y_{t}}{1+y_{t}} d_{t-1}+a\left(d_{t-1}-d^{*}\right)
$$

donde $p b^{\star}$ es el superávit primario que debería establecerse como objetivo para aproximar el stock de deuda pública al valor de referencia $d^{*}$ a una velocidad $a$. Expresándolo en términos de objetivos de déficit público $\left(d e f^{\star}\right)$, la expresión anterior quedaría como sigue:

$$
d e f^{*}=\frac{y_{t}}{1+y_{t}} d_{t-1}-a\left(d_{t-1}-d^{*}\right)
$$

A la hora de decidir entre las expresiones [2] y [3] optamos por la última. Ello es debido, en primer lugar, a la mayor visibilidad que aporta el concepto de déficit frente al del superávit primario. $Y$, en segundo lugar, queremos evitar la discusión en torno a los tipos de interés aplicables.

Esta metodología determinaría unos objetivos relativamente más exigentes para las comunidades autónomas más endeudadas. No obstante, tal y como se recoge con detalle en Martínez López y González (2020), los niveles de exigencia se pueden dosificar a través de la elección de los parámetros $d^{\star}$ y $a$, esto es, del nivel de referencia a efectos de la deuda pública y la velocidad de convergencia hacia el mismo, respectivamente. Por su parte, nuestra propuesta también está capacitada para recoger la situación de aquellas CC AA en las que una infrafinanciación relativa inferior a la media puede haber devenido parcialmente en un mayor endeudamiento público.

Aunque en la definición de los objetivos de déficit no se consideran los efectos del ciclo económico, la metodología sugerida sí cuenta con ello a la hora de evaluar el cumplimiento fiscal. Con otras palabras, para verificar si se cumplen los objetivos fiscales -definidos al margen de la posición cíclica de la economíasí se estudia el impacto que el componente coyuntural del saldo presupuestario ha podido ejercer en el resultado final. Así, razonando de manera simétrica en términos de saldo estructural, se penalizan los incumplimientos $\triangleright$ 
cometidos cuando en fases alcistas del ciclo se adoptan políticas fiscales expansivas. También, en sentido contrario, si el objetivo inicialmente establecido fuese demasiado exigente a la vista de la situación económica y la aparición de déficit coyuntural hubiese impedido la consecución del objetivo, la penalización debería atenuarse si no eliminarse.

Propuesta 2. Regla de gasto mejorada y una variable ancla ligada a la deuda

Nuestra segunda propuesta mantiene la preocupación por garantizar un proceso pausado de desendeudamiento, poniendo el énfasis en acomodar las reglas fiscales al ciclo económico y prestando una especial atención a la protección de la inversión pública. Ello pasaría por eliminar la exigencia de equilibrio estructural y las reglas de oro, para basar el sistema en una regla de gasto mejorada con la finalidad de converger a un nivel de deuda adaptado a los valores que encontraremos tras la crisis de la COVID-19.

El eje central de nuestra propuesta sería la fijación de una variable ancla (u objetivo final), que situaríamos en la deuda pública, y que a través de una variable instrumental como la regla de gasto se trataría de aproximar a un valor de referencia normativo. En efecto, siguiendo las conclusiones de Andrle et al. (2015), una regla de gasto definida convenientemente como un mecanismo reductor de la deuda presenta ciertas propiedades que permiten reducir la variabilidad del PIB durante la convergencia hacia el valor normativo de la variable ancla. No obstante, hay que reconocer que, con respecto a reglas basadas en equilibrios estructurales o nominales, este tipo de enfoques genera una mayor variabilidad del stock de deuda. Ello es debido a que la deuda refleja el mayor impacto de los estabilizadores automáticos y las propiedades contracíclicas de la regla de gasto que cuando se opta por reglas fiscales basadas en saldos presupuestarios nominales o estructurales.

De un modo general, una regla de gasto que pretendiese atender a esta doble perspectiva sería definida para el año t, $R G_{\mathrm{t}}$, en los términos que siguen:

$$
R G_{t}=F\left[a Y_{t}^{*}, \delta\left(D e b t_{t-1}-D e b t_{t}^{*}\right)\right]
$$

donde $Y_{t}^{*}$ es el output potencial y a la ponderación que este tiene en la definición de la regla de

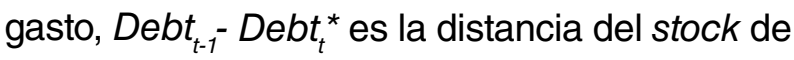
deuda del año anterior $D e b t_{t-1}$ al de referencia y normativo, denotado con un asterisco, siendo $\delta$ la ponderación que esta distancia recibe a la hora de calcular la regla de gasto. Según lo expuesto más arriba, la derivada parcial de F[.] con respecto al primer argumento sería positiva y con respecto al segundo, negativa. En este sentido, el primer argumento de F[.] recoge la preocupación por que la regla de gasto cuente con propiedades anticíclicas y el segundo atiende a los posibles problemas de sostenibilidad derivados del exceso de endeudamiento, ambos argumentos ponderados según se decida.

La aproximación que proponemos aquí, por tanto, conlleva un crecimiento del gasto real que sigue al del output potencial, al tiempo que no pierde de vista la necesaria consolidación fiscal para reducir el stock de deuda. La referencia del crecimiento del gasto al del output potencial obedece a diferentes razones, la principal de las cuales es dotarlo de propiedades de estabilización. Para simplificar la operativa, el concepto de output potencial podría sustituirse por el de output tendencial.

Es importante que seamos conscientes de que puede ser necesario adaptar esta nueva regla de gasto al contexto de los Gobiernos $\triangleright$ 
subnacionales. Así, aunque la variable deuda en relación con el PIB puede tener sentido en un ámbito nacional, en el caso de las CC AA y CC LL puede resultar más adecuada una referencia a los ingresos corrientes (Fernández y Lago, 2013). Por otra parte, sería necesario corregir no solo su diseño, sino también los problemas referidos con anterioridad respecto a la consolidación de incumplimientos pasados, la asincronía entre gastos e ingresos en los fondos recibidos de la UE o la interpretación de los aumentos permanentes de recaudación. Así, se deberían tener en cuenta los márgenes de cumplimiento o incumplimiento de la regla en un año concreto para determinar la tasa de crecimiento del gasto del año siguiente. Alternativamente, podría establecerse una misma tasa de referencia para varios años (que se podría revisar en el momento central de esos años), lo cual, además de evitar las consolidaciones, contribuiría a facilitar un ejercicio presupuestario a largo plazo, no teniendo que modificar las proyecciones cada vez que varía la tasa de referencia de años posteriores, situación que ocurre actualmente, dado que la tasa de referencia se va revisando cada año.

Según esta segunda propuesta, los incumplimientos de la regla de gasto darían lugar a planes económico-financieros tratando de asegurar el retorno a la senda de gasto coherente con el crecimiento de la economía. La no consolidación de incumplimientos o sobrecumplimientos permitiría una trayectoria de gasto a lo largo del tiempo coherente con la tasa de referencia, eliminando incentivos a un mayor gasto presente.

Así mismo, se podría abordar el problema originado por la regla de gasto en el ámbito de las CC LL y que ha provocado que éstas hayan restringido sus gastos y acumulado una elevada liquidez. En una futura reforma de la LOEPSF podría considerarse que la diferencia del menor gasto realizado sobre el que se podría realizar de acuerdo con la regla de gasto pudiera ser empleado en ejercicios posteriores para realizar inversiones o reformas estructurales (véase, por ejemplo, Ministerio de Hacienda de Suecia, 2017).

Por su parte, las reglas de oro quedarían sin efecto en nuestra propuesta por los problemas anteriormente citados. Sí sería conveniente mantener el principio de reducción de deuda pública cuando exista superávit o cuando se generen aumentos inesperados de ingresos, siendo necesario desarrollarlo reglamentariamente. Para ello resulta crucial centrarlo en el concepto de flujos de tesorería y condicionarlo a la inexistencia de costes asociados a la amortización, al tiempo que combinarlo con la regla de gasto anteriormente expuesta. En particular, esta podría ser modulada en función del nivel de endeudamiento para proteger el gasto público en inversión; así, se propone un esquema en el cual la posibilidad de realizar este gasto en inversión dependería del nivel de endeudamiento en relación con los ingresos corrientes, otorgando un nuevo incentivo a la reducción del nivel de endeudamiento o a la generación de ingresos.

De este modo, en los GSN cuyo nivel de endeudamiento fuera superior a un determinado porcentaje $D^{\star}$ de sus ingresos corrientes, el gasto público en inversión podría encajar con el cumplimiento de la regla de gasto definida en un horizonte plurianual. No obstante, sus inversiones financieramente sostenibles sí computarían en la regla de gasto. Para los GSN cuyo nivel de endeudamiento se encontrara entre un porcentaje $D^{*}-d$ de sus ingresos corrientes, el régimen actual de IFS les sería de aplicación, manteniéndose la verificación plurianual del gasto de inversión a efectos de la regla de gasto. $\mathrm{Y}$, finalmente, para los GSN cuyo endeudamiento fuera inferior a un $D$ 
porcentaje $d$ de sus ingresos corrientes, se permitiría que todo el gasto de inversión estuviera fuera del cómputo de la regla de gasto.

Además, para este último grupo de GSN con bajo nivel de endeudamiento, el margen de gasto no utilizado en un año hasta el cumplimiento con la regla de gasto se podría utilizar al año siguiente en gasto de inversión no computable a efectos de la nueva regla de gasto. Por su parte, el remanente de tesorería para gastos generales podría aplicarse a la realización de gasto de inversión, sin la obligatoriedad de reducir aún más el nivel de deuda, o al menos no en su totalidad, y dedicar el resto del remanente a la realización de inversión sin cómputo en la regla de gasto. De esta forma se reforzarían los incentivos positivos, circunstancia tan inusual en la normativa actual que pone más énfasis en los mecanismos sancionadores.

\section{Conclusión}

El actual marco normativo de reglas fiscales aplicable a Gobiernos subnacionales en España ha jugado un importante papel en la transformación de la gestión financiera pública de las Administraciones territoriales. Aunque su contribución a la consolidación fiscal de los últimos años sea discutida, ha aportado una mayor transparencia y control de las cuentas de los GSN que el marco anterior, reduciendo los riesgos que pueden afectar al conjunto del Reino de España. Asimismo, ha establecido un marco institucional de referencia alineado con las reformas de la gobernanza fiscal europea de principios de la década de 2010.

Ahora bien, la situación actual es muy distinta de cuando ese marco fue creado y la realidad pos-COVID-19 va a requerir cambios sustanciales en el mismo. Además, será necesario reenfocarlo al objetivo de reducción de deuda, que será la variable más importante en la política fiscal a partir de la recuperación económica. Además, existen otras reformas no menos importantes que deben simultanearse, si no anteceder, a la reforma de las reglas fiscales, a saber: la del sistema de financiación de los GSN y la de los mecanismos extraordinarios de financiación.

Este artículo ha evaluado el actual sistema de gobernanza fiscal español en lo que a los GSN se refiere. Así, ha prestado atención a cómo satisface las propiedades que la literatura exige a un sistema de reglas fiscales en un entorno descentralizado: efecto sobre la calidad de las finanzas públicas y capacidad para desarrollar políticas anticíclicas sin menoscabo de la sostenibilidad. También se han identificado los principales problemas de diseño de que adolece a nuestro juicio, y entre los que podríamos citar la coherencia entre reglas fiscales, la transparencia con que se aplica, la protección de la inversión pública y el acceso al endeudamiento.

A partir de esta valoración, hemos esbozado los rasgos generales de dos propuestas que podrían servir de base para una futura reforma del sistema de reglas fiscales de los GSN en nuestro país. Ambas propuestas comparten su preocupación por reducir el nivel de endeudamiento con que saldremos de la crisis sanitaria y económica provocada por la COVID-19. Pero no descuidan otras dimensiones como la economía política de los cambios, la evaluación de su cumplimiento (y posibles sanciones en su caso) o las extensiones hacia el gasto público en inversión, entre otras.

\section{Bibliografía}

AIReF, Autoridad Independiente de Responsabilidad Fiscal (2017). Informe de los Proyectos y Líneas $D$ 
fundamentales de los presupuestos de las Administraciones Públicas: Corporaciones Locales 2017.

AIReF, Autoridad Independiente de Responsabilidad Fiscal (2018). Informe 37/2018 de cumplimiento esperado de los objetivos de estabilidad presupuestaria, deuda pública y regla de gasto en 2018. https://www.airef.es/wp-content/uploads/2018/ 08/2018-07-25-Informe-prevision-de-cierre-2018180723-2.pdf

AIReF, Autoridad Independiente de Responsabilidad Fiscal (2020). Informe sobre la actualización del programa de estabilidad 2020-2021. Informe 2/2020. https://www.airef.es/wp-content/uploads/ 2020/05/Informe-APE/FINALInforme_APE2020-2021.pdf

Andrle, M., Bluedorn, M. J. C., Eyraud, L., Kinda, M. T., Brooks, P. K., Schwartz, M. G., y Weber, A. (2015). Reforming fiscal governance in the European Union. (I M F Staff Discussion Note, 15-19). International Monetary Fund. https://www.imf. org/external/pubs/ft/sdn/2015/sdn1509.pdf

Blanchard, O., Leandro, A., y Zettelmeyer, J. (2020). Redesigning the EU Fiscal Rules: From Rules to Standards. 72nd Economic Policy Panel Meeting. Federal Ministry of Finance, Germany. https://www.economic-policy.org/wp-content/ uploads/2020/10/9100_Redesigning-EU-Fiscal-Rules.pdf

Calvo, S., y Cadaval, M. (2021). The Impact of Soft Budget Constraint on the Fiscal Co-responsibility of the Autonomous Communities in Spain:The Case of Extraordinary Liquidity Funds (20122019). Hacienda Pública Española/Review of Public Economics. https://hpe-rpe.org/ief/109/ forthcoming-articles/4709/the-impact-of-softbudget-constraint-on-the-fiscal-co-responsibility-ofthe-autonomous-communities-in-spain-the-case-of-extraordinary-liquidity-funds-2012-2019.pdf

Comisión Europea (2020). Economic governance review. https://ec.europa.eu/info/business-economyeuro/economic-and-fiscal-policy-coordination/ eu-economic-governance-monitoring-prevention-correction/economic-governance-review_en
Cuenca, A. (2015). Las comunidades autónomas en 2015: estabilidad presupuestaria y sostenibilidad financiera. Cuadernos de Información Económica (246), 47-58.

Delgado-Téllez, M., Kataryniuk, I., Pérez, J.J., y Gordo, E. G. (2020). The decline in public investment: "social dominance» or too-rigid fiscal rules? (Documentos de trabajo del Banco de España, 2025). https://www.bde.es///webbde/SES/ Secciones/Publicaciones/PublicacionesSeriadas/DocumentosTrabajo/20/Files/dt2025e.pdf

Delgado-Téllez, M., Duarte Lledó, V., y Pérez, J.J. (2017). On the Determinants of Fiscal Non-Compliance: An Empirical Analysis of Spain's Regions. (IMF Working Papers 2017/005). https://www.imf. org/en/Publications/WP/Issues/2017/01/20/ On-the-Determinants-of-Fiscal-Non-ComplianceAn-Empirical-Analysis-of-Spains-Regions-44552

Dovis, A., y Kirpalani, R. (2020). Fiscal rules, bailouts, and reputation in federal governments. American Economic Review, 110(3), 860-88.

European Fiscal Board (2019). Annual Report 2019 of the European Fiscal Board. Secretariat of the European Fiscal Board, European Commission.

Eyraud, L., Debrun, X., Hodge, A. , Duarte Lledó, V., y Pattillo, C. A. (2018). Second-Generation Fiscal Rules: Balancing Simplicity, Flexibility, and Enforceability. (IMF Staff Discussion Notes 18/04). https://www.imf.org/en/Publications/Staff-Discussion-Notes/Issues/2018/04/12/SecondGeneration-Fiscal-Rules-Balancing-Simplicity-Flexibility-and-Enforceability-45131

Eyraud, L., Hodge, A., Ralyea, J., y Reynaud, J. (2020). How to Design Subnational Fiscal Rules: A Primer. (IMF How to Notes). https://www.imf. org/en/Publications/Fiscal-Affairs-DepartmentHow-To-Notes/lssues/2020/02/25/How-to-DesignSubnational-Fiscal-Rules-A-Primer-48967

Fernández Llera, R. (2016). Control del endeudamiento autonómico y estabilidad presupuestaria: Evolución y propuestas de futuro. Revista de Estudios Regionales, (105), 103-136. 
Fernández, X., y Lago, S. (2013). Sobre el reparto de los derechos de déficit entre comunidades autónomas: una propuesta alternativa. Revista de Economía Aplicada, (63), 117-136.

Gadea, M. D., Gómez-Loscos, A., y Montañés, A. (2012). Cycles inside cycles: Spanish regional aggregation. SERIEs, 3(4). 423-456.

Hauptmeier, S., y Kamps, C. (2020). Debt Rule Design in Theory and Practice: The SGP's Debt Benchmark Revisited. (ECB Working Paper, 2379). https://papers.ssrn.com/sol3/papers.cfm? abstract_id=3548577

Hernández de Cos, P. (2020). La economía española ante la crisis del COVID-19. Comparecencia ante la Comisión de Asuntos Económicos y Transformación Digital del Congreso de los Diputados, 18 de mayo de 2020. (Documentos ocasionales/Banco de España, 2023). https:// www.bde.es/f/webbde/SES/Secciones/Publicaciones/PublicacionesSeriadas/DocumentosOcasionales/20/Fich/do2023.pdf

Instituto de Estudios Fiscales (2018). Reforma de la Financiación Territorial: Informes de las Comisiones de Expertos de 2017. Secretaría de Estado de Hacienda, Madrid. https://www.ief.es/docs/ destacados/publicaciones/libros/lb/2018_ReformaFinanciacionTerritorial.pdf

Jimeno, J. F., y Martínez López, D. (10 de noviembre de 2020). Presupuestos históricos (e histéricos). Nada es Gratis. https://nadaesgratis.es/juan-francisco-jimeno/presupuestos-historicos-e-histericos

Lago, S. (2017) (coord.). Estabilidad presupuestaria y sostenibilidad financiera de las Comunidades Autónomas.(GEN+Report, 4/2017). Consellería de Facenda. Xunta de Galicia. https://www.researchgate.net/publication/320893897_ Estabilidad_presupuestaria_y_sostenibilidad_financiera_de_las_Comunidades_Autonomas

Martínez López, D. (2007). La nueva ley de estabilidad presupuestaria: implicaciones para las Comunidades Autónomas. En S. Lago (ed.), La Financiación del Estado de las Autonomías: Perspectivas de Futuro (pp. 389-414). Instituto de Estudios Fiscales.
Martínez López, D. (23 de marzo de 2020). Apuntes de federalismo fiscal sobre el COVID-19. Nada es Gratis.https://nadaesgratis.es/admin/apuntesde-federalismo-fiscal-sobre-el-covid-19

Martínez López, D. (2020b). La gobernanza fiscal de las Comunidades Autónomas. Una valoración crítica de su estado actual con perspectivas de reforma. Investigaciones Regionales-Journal of Regional Research (47), 31-56.

Martínez López, D. (2021). Subnational borrowing and bailouts: When the federal government looks at the votes (differently) and its borrowing matters. (GEN Working Paper A2021-1).

Martínez López, D., y González, F. (2020). Los objetivos de déficit en las CC AA: Una propuesta basada en la deuda. (Fedea Policy Papers 2020-21). https://documentos.fedea.net/pubs/fpp/2020/10/ FPP2020-21.pdf

Ministerio de Hacienda. Secretaría General de Financiación Autonómica y Local (2020a). Informe sobre los mecanismos de financiación de CC AA, Balance 2012-2019. https://www.hacienda.gob. es/CDI/Mecanismos\%20Financiaci\%C3\%B3n/ Informe_Balance_Mecanismos_2012-2019.pdf

Ministerio de Hacienda. Secretaría General de Financiación Autonómica y Local (2020b). Informe sobre los mecanismos de financiación de las Entidades Locales.

Ministerio de Hacienda de Suecia (2017). The Swedish fiscal policy framework. Comunicación del Gobierno al Parlamento 2017/18:207. https:// www.government.se/49feed/globalassets/gover$\mathrm{nment} /$ dokument/finansdepartementet/the-swedish-fiscal--policy-framework.pdf

Molina Parra, A., y Martínez López, D. (2018). Do federal deficits motivate regional fiscal (im)balances? Evidence for the Spanish case. Journal of Regional Science, 58(1), 224-258.

OECD/United Cities and Local Governments (2016). Subnational Governments Around the World: Structure and Finance. OECD Publishing. https://www.uclg.org/sites/default/files/ global_observatory_of_local_finance_part_i-ii. pdf 
